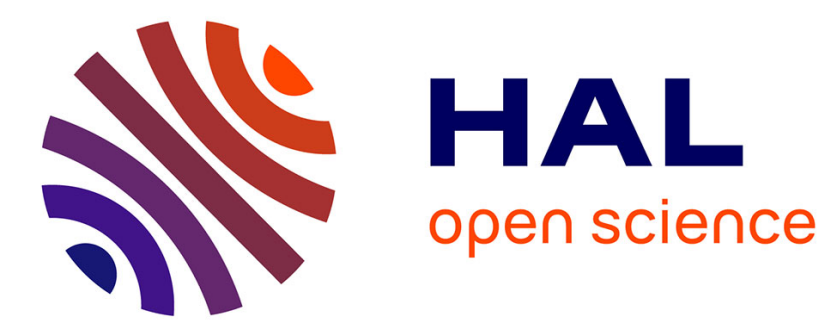

\title{
Copper hexacyanoferrate functionalized singlewalled carbon nanotubes for selective cesium extraction
}

\author{
H. Draouil, Laurent Alvarez, J. Causse, V. Flaud, M. A. Zaibi, Jean-Louis
}

Bantignies, M. Oueslati, J. Cambedouzou

\section{- To cite this version:}

H. Draouil, Laurent Alvarez, J. Causse, V. Flaud, M. A. Zaibi, et al.. Copper hexacyanoferrate functionalized singlewalled carbon nanotubes for selective cesium extraction. New Journal of Chemistry, 2017, 41 (15), pp.7705-7713. 10.1039/c7nj00879a . hal-01576846

\section{HAL Id: hal-01576846 \\ https://hal.science/hal-01576846}

Submitted on 7 Oct 2020

HAL is a multi-disciplinary open access archive for the deposit and dissemination of scientific research documents, whether they are published or not. The documents may come from teaching and research institutions in France or abroad, or from public or private research centers.
L'archive ouverte pluridisciplinaire HAL, est destinée au dépôt et à la diffusion de documents scientifiques de niveau recherche, publiés ou non, émanant des établissements d'enseignement et de recherche français ou étrangers, des laboratoires publics ou privés. 


\title{
Copper hexacyanoferrate functionalized single-walled carbon nanotubes for selective cesium extraction
}

\author{
H. Draouil ${ }^{1,2,3,4}$, L. Alvarez ${ }^{4}$, J. Causse ${ }^{1}$, V . Flaud ${ }^{5}$, M.A. Zaïbi ${ }^{3}$, J.L. Bantignies ${ }^{4}$, M. Oueslati ${ }^{2}$ and J. \\ Cambedouzou ${ }^{1}$. \\ ${ }^{1}$ Institut de Chimie Séparative de Marcoule, UMR 5257 CEA-CNRS-ENSCM-UM, BP17171, F-30207 Bagnols \\ sur Cèze, France \\ ${ }^{2}$ Unité de nanomatériaux et photonique, Université El Manar, Faculté des Sciences de Tunis, Département de \\ Physique, 2092 El Manar, Tunis Tunisia \\ ${ }^{3}$ Ecole Nationale Supérieure d'Ingénieurs de Tunis, Université de Tunis, 5 Avenue Taha Hussein, 1008 Tunis, \\ Tunisia \\ ${ }^{4}$ Laboratoire Charles Coulomb (L2C), UMR 5221 CNRS, Université de Montpellier, F-34000 Montpellier, France \\ ${ }^{5}$ Institut Charles Gerhardt Montpellier, UMR5253, Université de Montpellier, F-34000 Montpellier, France
}

\begin{abstract}
Single-walled carbon nanotubes (SWCNTs) are functionalized with copper hexacyanoferrate (CuHCF) nanoparticles to prepare solid substrates for the sorption of cesium ions $\left(\mathrm{Cs}^{+}\right)$from liquid outflows. The high mechanical resistance and large electrical conductivity of SWCNTs are associated to the ability of CuHCF nanoparticles to selectively complex $\mathrm{Cs}^{+}$ions in order to achieve membrane-like buckypapers presenting high loading capacity of cesium. The materials are thoroughly characterized using electron microscopy, Raman scattering, X-ray photoelectron spectroscopy and thermogravimetric analyses. Cs sorption isotherms are plotted after having measured the $\mathrm{Cs}^{+}$concentration by liquid phase ionic chromatography in the solution before and after exposure to the materials. It is found that the total sorption capacity of the material reaches $230 \mathrm{mg} \cdot \mathrm{g}^{-1}$, and that about one third of the sorbed Cs $\left(80 \mathrm{mg} \cdot \mathrm{g}^{-1}\right)$ is selectively complexed in the CuHCF nanoparticles grafted on SWCNTs. These high values open interesting outlooks in the integration of such materials in devices for the controlled sorption and desorption of these ions.
\end{abstract}

\section{Introduction}

Radiotoxic elements constitute a source of pollution from the nuclear industry, for which it is important to anticipate effective processes. Among the elements of high radiotoxicity, ${ }^{137} \mathrm{Cs}$ constitutes a priority since it possesses a long half-life of more than 30 years and it decays to ${ }^{137 \mathrm{~m}} \mathrm{Ba}$ presenting a high gamma emission of $0.662 \mathrm{MeV}$. It has furthermore been released in former nuclear accidents and it has become an urgency to provide perennial solutions for treating water contaminated by radioactive cesium ions $\left(\mathrm{Cs}^{+}\right)$. Up to now, several materials have been proposed to achieve this application. However, the frequent coexistence of other ions in much larger concentration than $\mathrm{Cs}^{+}$in contaminated water makes the use of selective sorbents necessary in order to achieve efficient cleaning. Among the most promising materials envisioned for this application, powders of analogous of Prussian blue have been shown to selectively uptake large quantities of $\mathrm{Cs}^{+}$ions [1-2]. However, this material tends to form sludge when hydrated, therefore complicating the process and the waste elimination. 
Extraction on solid support is an interesting option because it allows treating large quantities of effluent by continuous processes, and to trap radioactive substances into more easily manipulable solid matrices. Attempts to graft Prussian blue analogues on solid supports such as mesoporous silica were made in the recent years [3-4], and showed that it was possible to benefit from the sorption capacity of Prussian blue and, at the same time, to get a material easy to handle. However, silica based materials present an inherent weakness in their chemical composition: they are intolerant to basic medium, which restricts their application field. Singlewalled carbon nanotubes (SWCNTs) based membranes seem to be effective materials for this application as they combine several advantages. First, they often present large specific surface areas, considering that they can uptake important amounts of cesium. Moreover, the atomic structure of SWCNTs is chemically stable and resistant even to aggressive environments such as acidic or basic media.

As a matter of facts, it turned out from the literature that carbon nanotubes are good candidates for the sorption of toxic chemical elements and of many heavy metal ions such as $\mathrm{Cs}^{+}, \mathrm{Cd}^{2+}$, $\mathrm{Pb}^{2+}, \mathrm{Sr}^{2+}$ [5-9]. Yavari et al [5] first oxidized multi walled carbon nanotubes (MWCNTs) by nitric acid. They successfully grown functional groups such as carboxylic, lactone and phenol on MWCNTs surface. They reported that the amount of $\mathrm{Cs}^{+}$uptaken by functionalized MWCNTs depends upon aqueous solution parameters such as ionic strength, $\mathrm{pH}$, and concentration of $\mathrm{Cs}^{+}$. The highest adsorption of $\mathrm{Cs}^{+}$by functionalized MWCNTs was found at higher $\mathrm{pH}$, lower ionic strength and lower initial concentration of $\mathrm{Cs}^{+}$in aqueous solution. A. K. Vinin et al [6] studied the removal of $\mathrm{Cs}^{+}$and $\mathrm{Sr}^{2+}$ from aqueous solution by MWCNTs dispersed in sodium cobalt hexacyanoferrate (CoHCF). They reported that the presence of MWCNTs enhances the adsorption of $\mathrm{Cs}^{+}$and $\mathrm{Sr}^{2+}$ on CoHCF. Recently, the same authors [7] have exchanged CoHFC by zeolite-A, which is encapsulated into MWCNTs. They have found that the quantity of removed $\mathrm{Cs}^{+}$decreased, but that the $\mathrm{Sr}^{2+}$ extraction was favored. In order to further improve the performance of these materials for ionic sorption, it is interesting to use the known and mastered chemistry of carbon nanotubes (CNTs) in order to functionalize them with molecules or nanoparticles able to specifically capture the targeted ions. Some works have been carried out in this view using MWCNTs as support for CuHCF-NPs [10-11], and a Cs ${ }^{+}$sorption capacity of about $150 \mathrm{mg} \cdot \mathrm{g}^{-1}$ was found for this substrate. Provided that SWCNTs possess a higher specific surface area than MWCNTs, they can be considered as a promising solid support for functionalization with molecules able to capture cations. Our group recently demonstrated that it is possible to graft dimethyl-acetylene dicarboxylate (DMAD) [9] and other organic functions [12] by a simple protocol based on Diels-Alder (DA) cycloadditions. In the case of DMAD grafted SWCNTs, we reported a high capacity of $\mathrm{Cs}^{+}$capture $(250 \mathrm{mg}$ of Cs per gram of grafted SWCNTs) [9]. These molecules are however not selective for $\mathrm{Cs}^{+}$sorption, and we therefore considered functionalization by selective moieties.

In this work, we present an efficient method to functionalize SWCNTs with copper hexacyanoferrate nanoparticles (CuHCF-NPs). We indeed aim to combine the ability to selectively capture $\mathrm{Cs}^{+}$ions of HCFs with the exceptional mechanical and electronic properties of SWCNT to elaborate efficient and reusable devices for the removal of large quantities of $\mathrm{Cs}^{+}$. We use a simple and original protocol based on two steps: first the functionalization of SWCNTs with propargylamine $\mathrm{C}_{3} \mathrm{H}_{5} \mathrm{~N}_{1}$ (PRG), which is a primary amine used as a bridge between the SWCNT and CuHCF-NPs. The second step is the growth of CuHCF nanoparticles 
from the grafted PRG. This phase is based on two reactions: firstly the addition of copper nitrate $\mathrm{Cu}\left(\mathrm{NO}_{3}\right)_{2}$ and hence the addition of potassium ferrocyanide $\mathrm{K}_{4} \mathrm{Fe}(\mathrm{CN})_{6}$. These insertions are repeated for three cycles.

We therefore present the characterization of the functionalized samples after each step of the elaboration procedure using different characterization techniques. The functionalization rate is quantified by thermogravimetric analysis (TGA) combined with spectroscopic analyzes including Raman spectroscopy and X-ray photoemission spectroscopy (XPS). High resolution transmission electron microscopy (HRTEM) is used to follow the evolution of the grafted nanoparticles growth.

Finally, we detail the $\mathrm{Cs}^{+}$sorption capacity with both raw SWCNTs and CuHCF-NPs functionalized SWCNTs. Based on measurements of $\mathrm{Cs}^{+}$concentration before and after exposure to CuHCF-NPs functionalized SWCNTs by liquid phase ionic chromatography (LPIC), we obtained $\mathrm{Cs}^{+}$sorption isotherms showing a total $\mathrm{Cs}^{+}$sorption capacity of about 230 mg. $\mathrm{g}^{-1}$, and a selective sorption capacity of about $80 \mathrm{mg}$ of $\mathrm{Cs}^{+}$per gram of grafted SWCNTs. This removal capacity compares to the highest efficiencies measured on other substrates, which is very encouraging in the perspective of developing reversible devices for the decontamination of liquids.

\section{Experimental section}

\section{Sample preparation}

SWCNTs were purchased from Carbon Solution, Inc.(P2-SWCNTs). The diameter distribution is $1.4 \pm 0.2 \mathrm{~nm})$. The carbon purity of our SWCNTs is higher than $90 \mathrm{wt} . \%$ and the metal $(\mathrm{Ni}, \mathrm{Y})$ content is between 4-8 wt.\%. Propargylamine (PRG), $\mathrm{Cu}\left(\mathrm{NO}_{3}\right)_{2}$ and $\mathrm{K}_{4} \mathrm{Fe}(\mathrm{CN})_{6}$ reactants were purchased from Sigma-Aldrich (99\%) and used without further purification. The functionalization of SWCNTs by CuHCF-NPs was realized following two steps:

\section{1- Functionalization with propargylamine}

In the first step, we start from a mixture containing 60mg of SWCNTs with $25 \mathrm{ml}$ of pure PRG. To optimally disperse SWCNTs in PRG, the mixture is ultrasonicated for $15 \mathrm{~min}$ following the procedure described elsewhere [9]. The mixture is then transferred into a teflon-lined steel autoclave and heated to $100^{\circ} \mathrm{C}$ during 24 hours in order to allow the Diels Alder reaction between PRG and SWCNTs (Fig. 1a). This mixture is filtered and then washed with acetone in order to remove PRG molecules that did not covalently bound to SWCNTs. It is finally dried at room temperature. The recovered sample consists of a easy-to-handle buckypaper of grafted SWNCTs.

\section{2-Cu-HFC-NPs growth}

This step is based on two reactions, following the procedure described in a previous work [13]. The first step features the addition of a copper nitrate $\mathrm{Cu}\left(\mathrm{NO}_{3}\right)_{2}$ solution in order to attach a $\mathrm{Cu}$ atom to the PRG. This $\mathrm{Cu}$ atom will serve as a nucleation center for the further growth of the CuHCF-NP. After careful rinsing with acetone, the second step consists in the addition of a potassium ferrocyanide $\mathrm{K}_{4} \mathrm{Fe}(\mathrm{CN})_{6}$ solution aiming at initiating the growth of the first cell of 
the CuHCF-NP, as depicted in Fig. 1b. Both solutions are prepared with a concentration of $10^{-}$ ${ }^{2} \mathrm{M}$. The reactions are performed at room temperature, under simple agitation, and repeated for three cycles. The first cycle lasts $2 * 24$ hours, the second one lasts $2 * 12$ hours and the third one lasts $2 * 8$ hours. The solution is then filtered, and the recovered sample is carefully washed with acetone, and dried at room temperature for 48 hours. When immersed in a solution containing $\mathrm{Cs}^{+}$ions, the $\mathrm{K}^{+}$anions are expected to be replaced by the $\mathrm{Cs}^{+}$anions initially in solution. This mechanism of ionic exchange is depicted in Fig.1.c.
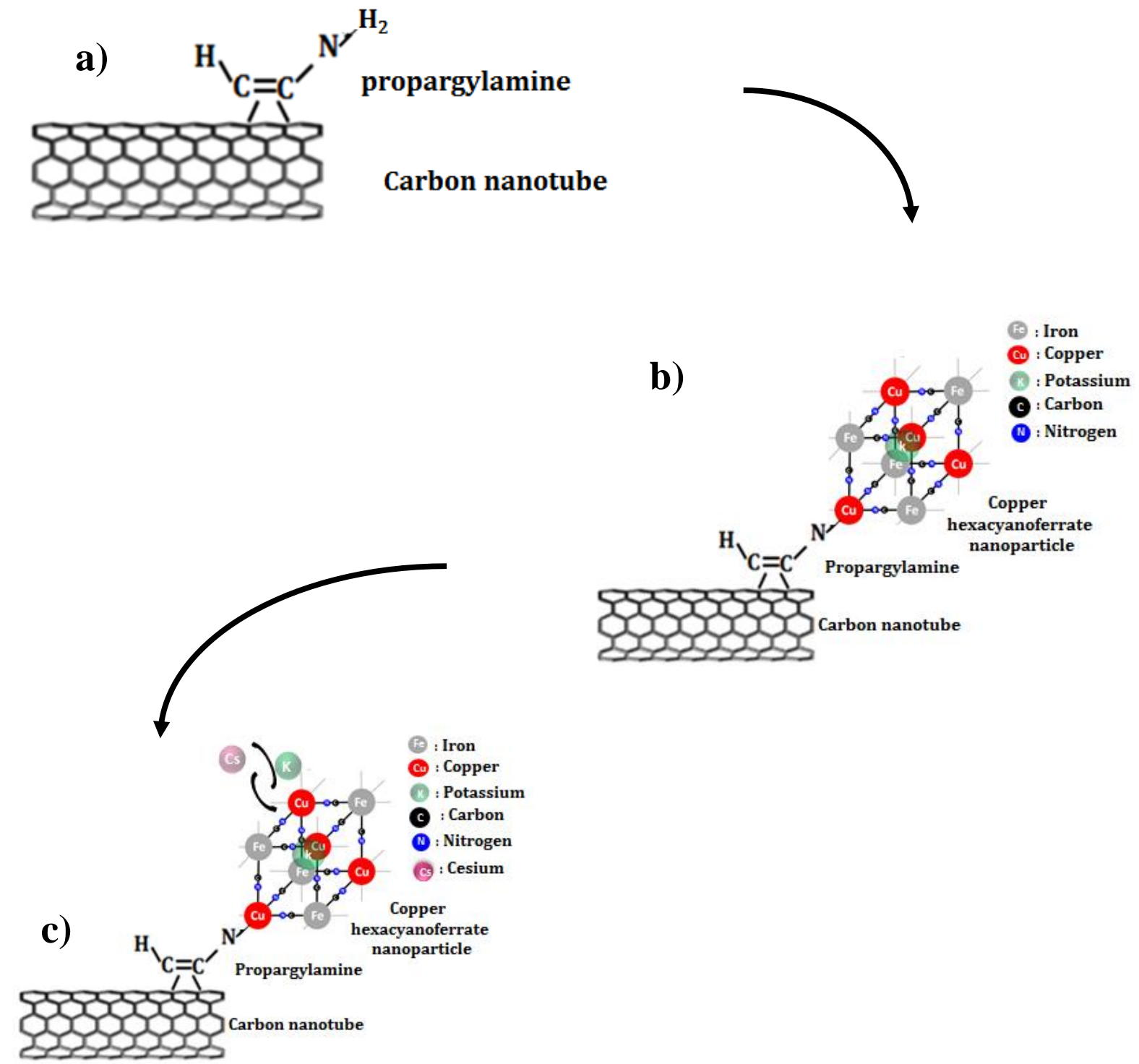

Figure 1: Schematic representations of a) SWCNT functionalized with propargylamine, b) SWCNT functionalized with a HCF-NP, and c) Exchange mechanism between $\mathrm{K}^{+}$and $\mathrm{Cs}^{+}$ ion.

\section{Material characterization}

The chemical composition of the materials is monitored by X-ray photoelectron spectroscopy (XPS) on an ESCALAB 250 (Thermo Electron). The X-ray excitation is provided by a monochromatic Al Ka (1486.6eV) source. Analyzed surface has a $400 \mu \mathrm{m}$ diameter. All XPS 
spectra were treated using AVANTAGE software. The background signal was removed using the Shirley [14] method. Surface atomic concentrations are determined from photoelectron peaks areas using the atomic sensitivity factors reported by Scofield [15]. During the measurements the base pressure in the analysis chamber is around $8 \times 10^{-10}$ Torr. Binding energies (BE) of all core levels are referred to the $\mathrm{C}=\mathrm{C}$ of $\mathrm{C} 1 \mathrm{~s}$ carbon at $284.4 \mathrm{eV}$.

The functionalization rate is deduced by thermogravimetric analyses (TGA), which are performed on a Setaram SETSYS evolution TGA/TDA 16 under Argon atmosphere with a heating rate of $5^{\circ} \mathrm{C} / \mathrm{min}$.

Raman scattering experiments are carried out on a Horiba Jobin Yvon LabRAM Aramis spectrometer. Two excitation laser wavelengths are used: (i) $\lambda=633 \mathrm{~nm}$ with a spot size of $1 \mu \mathrm{m}$, a $\times 100$ objective, the recording time is set equal to $2 \times 30 \mathrm{~s}$ and power is kept at $610 \mu \mathrm{W}$, (ii) $\lambda=$ $532 \mathrm{~nm}$ with a spot size of $1 \mu \mathrm{m}$, a $\times 50$ objective, the power is kept at $475 \mu \mathrm{W}$ and the recording time is set equal to $2 \times 50$ s. Other Raman experiments are performed using a T64000 Spectrometer Raman Jobin Yvon, where the excitation laser wavelength is $488 \mathrm{~nm}$ with a $x 50$ objective, the power is kept at $620 \mu \mathrm{W}$ and the recording time is set equal to $2 \mathrm{x} 40 \mathrm{~s}$.

Transmission electron microscopy (TEM) is performed on a JEOL 2200FS. Samples are deposited on 400-mesh holey carbon-coated copper grids.

Liquid phase ionic chromatography (LPIC) is performed using an ICS-5000-Dionex instrument with 4 scans for each sample.

\section{Cesium sorption tests}

$\mathrm{Cs}^{+}$sorption measurements are carried out in batch mode on both raw SWCNTs and on CuHCFNPs functionalized SWCNTs according to the following protocol: 10mg of a buckypaper of carbon nanotubes are stirred with $20 \mathrm{ml}$ of a cesium nitrate solution using different concentrations $(0.1 \mathrm{mM}, 0.5 \mathrm{mM}, 2 \mathrm{mM}, 4 \mathrm{mM}$ and $8 \mathrm{mM})$ for $24 \mathrm{~h}$. Carbon nanotubes are recovered by filtration using a filter paper with particle retention between 5 and $13 \mu \mathrm{m}$ and thoroughly washed using Milli-Q water. The solid samples are then dried for further analyses, while the liquid phase is analyzed by LPIC. The LPIC measurements of the solutions before and after exposure to carbon nanotubes allowed us to plot the sorption isotherm of $\mathrm{Cs}^{+}$.

\section{Results and Discussion}

In order to characterize the structure of SWCNTs after functionalization with CuHCF-NPs, we performed a multi-method analysis of the sample before and after the functionalization protocol.

\section{High Resolution Transmission Electron Microscopy}



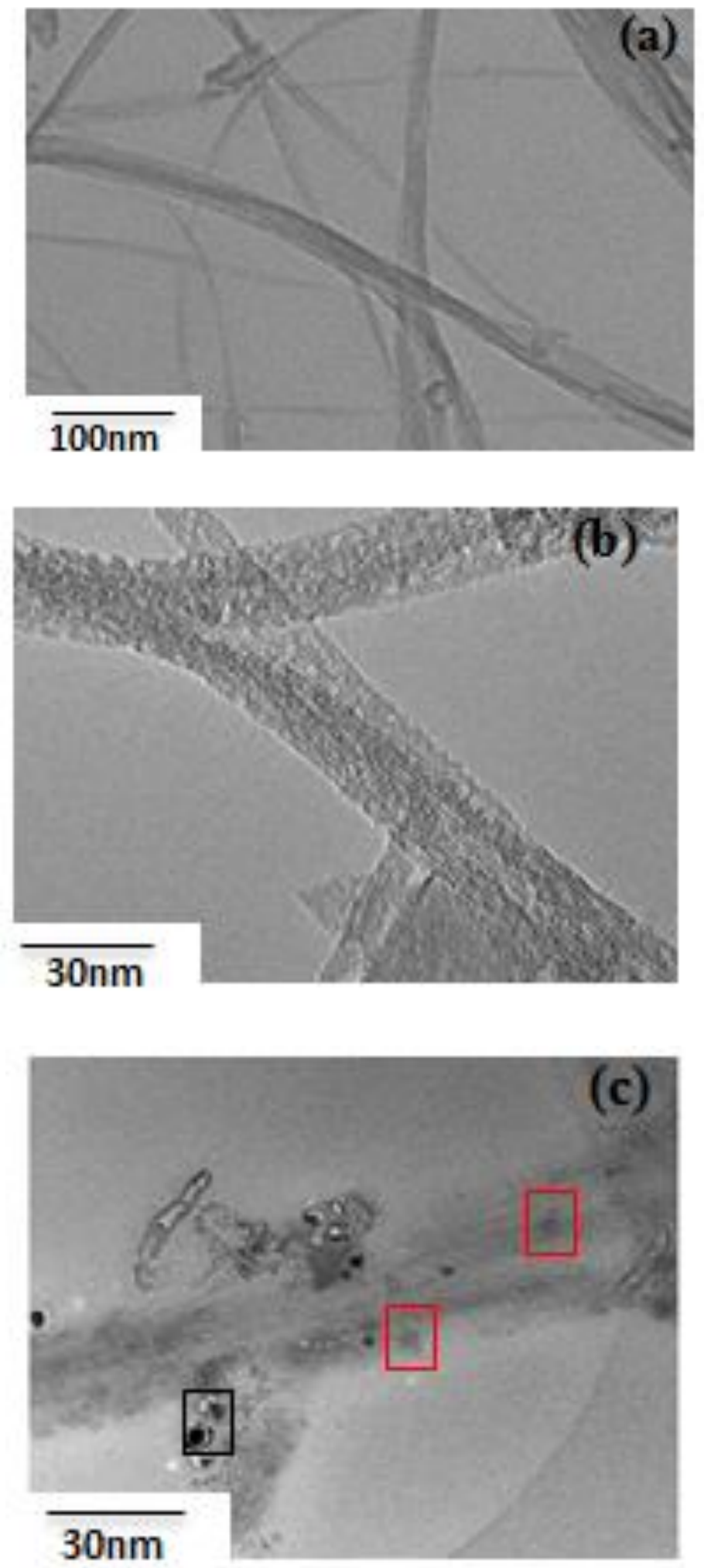

Figure 2: HRTEM images of raw SWCNT at low and high magnification (a and b, respectively) and SWCNTs-CuHCF (c). Black squares and red squares magnify additional features with regards to raw SWCNTs. 
HRTEM images of pristine SWCNTs are shown in Fig. 2.a at low magnification. Clean bundles of SWCNTs are seen in the sample. On the higher magnification image shown on Figure 2.b, the bundles appear slightly distorted, probably due to the acceleration voltage of $200 \mathrm{kV}$, but their surface have still a clean aspect. The length of these bundles is estimated to be superior to $1 \mu \mathrm{m}$. After functionalization, the TEM image (Fig. 2.c) shows that the sample becomes decorated by some particles presenting a typical size of a few nanometers and distributed throughout the nanotube bundle. Some of these particles appear darker (black squares in Fig. 2.c), however the majority of the NPs appears in light grey (red squares in Fig. 2.c) and are likely to be CuHCF-NPs. The difference in contrast could be due to the distance of the objects with regard to the focalization plane of electrons. In order to prove the chemical nature of the NPs, we performed complementary characterizations that are provided below.

\section{Thermogravimetric analysis}

The rate of PRG and CuHCF grafted onto the SWCNTs surface was determined by TGA measurements. TGA curves of raw SWCNTs, SWCNTS_PRG and SWCNTs_CuHCF are shown in Fig. 3.a, b and c, respectively.

The weight loss observed below $100^{\circ} \mathrm{C}$ in raw SWCNTs (about $2 \mathrm{wt}$. \%) is related to the desorption of water or ungrafted molecules from SWCNTs surface. A more important weight loss is then observed in the temperature region between $250^{\circ} \mathrm{C}$ and $600^{\circ} \mathrm{C}$, almost in the same proportion for both SWCNTs_PRG and SWCNTs_CuHCF. This temperature range corresponds to the elimination of PRG moieties [12]. Above $600^{\circ} \mathrm{C}, \mathrm{SWCNTs}$ _PRG shows the same weight loss than pristine SWCNTs. However, a supplemental weight loss is observed in the case of SWCNTs_CuHCF on a large temperature range, which could be related to the thermal degradation of the organic $(\mathrm{C})$ and inorganic $(\mathrm{Cu}, \mathrm{Fe}, \mathrm{K}$ and $\mathrm{N})$ moieties found in CuHCF nanoparticles [11]. Considering that the amount of PRG molecules and CuHCF- NPs grafted on carbon nanotubes is calculated based on the differential weight loss with regards to pristine SWCNTs, the PRG functionalization rate is estimated to $12 \mathrm{wt} . \%$, which corresponds to 1 PRG grafted molecule per 33 carbon atoms, and the proportion of CuHCF_NPs is estimated to about $15 \mathrm{wt}$. \%. These obtained grafting rates are in the same order as for several recently reported functionalized carbon nanotubes [9, 16-17]. 


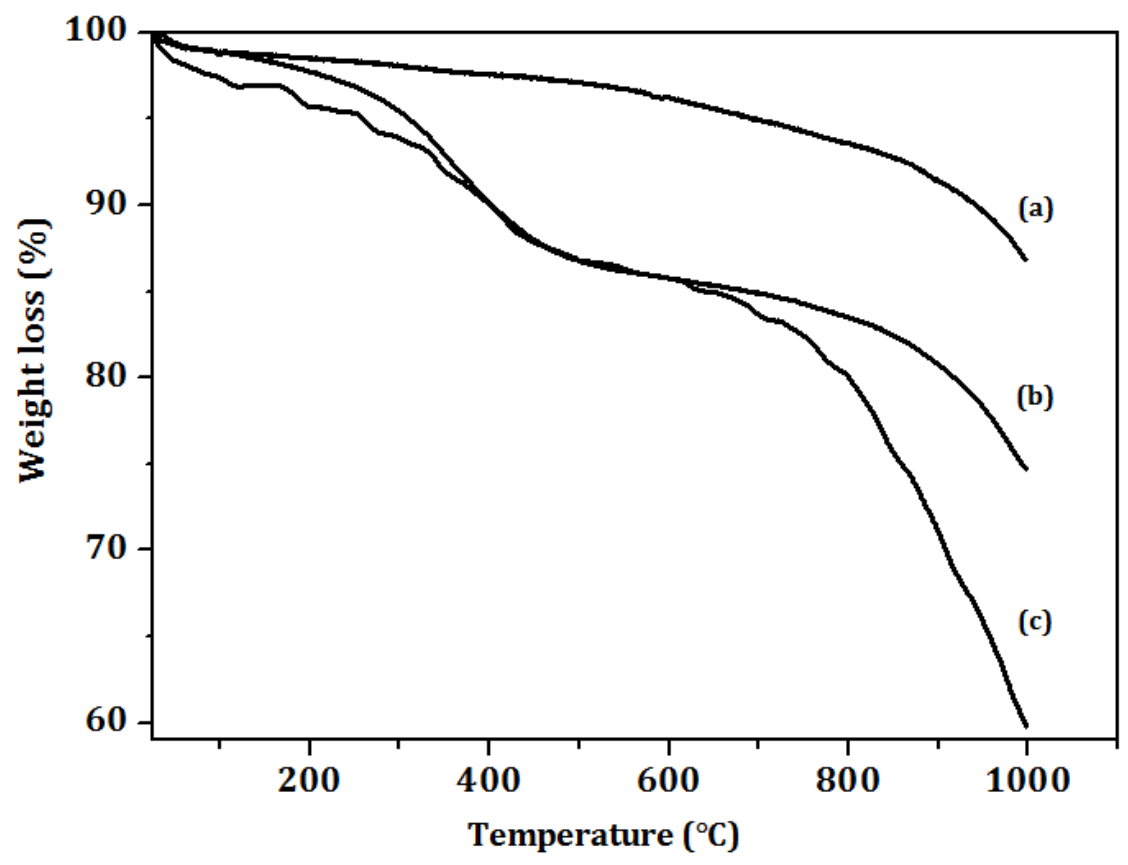

Figure 3: TGA measured under argon atmosphere of a) raw SWCNT; b) SWCNTs_PRG and c) SWCNTs_CuHCF

\section{Raman spectroscopy}

Raman spectra were recorded at room temperature on raw SWCNTs as well as on modified samples. In order to check the homogeneity of the functionalization, all treated samples were analyzed by Raman spectroscopy on 10 different regions. Very similar signals were found on each area, confirming the homogeneity of PRG and CuHCF_NPs grafting onto the SWCNTs surface.

For Raman analyses measured with an excitation wavelength of $633 \mathrm{~nm}$, the spectra of functionalized samples shows distinct changes from that of raw SWCNTs (Fig. 4). The signals originating from SWCNTs Radial Breathing Modes (RBM) appear between 100 and $200 \mathrm{~cm}^{-1}$ depending on the tube diameter. We observe a strong decrease of the RBM intensity of modified samples (RBM band in Fig. 4). These changes are typical of chemical alteration of SWCNTs, and we indeed believe that the presence of PRG and CuHCF_NPs bound on SWCNTs surface impacts the normal breathing of the tubes. Similar effects have been reported by S. Hussain et al [18], who have investigated modified SWCNTs and observed that all RBM intensities of treated samples decreased and disappeared, in relation with the duration of the acid treatment inflicted to SWCNTs. Also, R. Liu [19] found that 6 hours of sonication of SWCNTs causes a decrease of high wavenumber RBM intensities and explain it by the destruction of the tubes of small diameter or reduction in resonance enrichment of functionalized SWCNTs.

In the higher wavenumber area near $\sim 1590 \mathrm{~cm}^{-1}$, we observe the tangential $\mathrm{G}$ modes of SWCNTs (G band in Fig. 4). With a 633nm excitation wavelength for Raman analyses we observe a Breit-Wigner-Fano (BWF) lineshape at around $1540 \mathrm{~cm}^{-1}$ originating from the electron-phonon coupling in metallic nanotubes [20]. The BWF signal completely disappears for SWCNTs_PRG as well as for SWCNTs_CuHCF. This disappearance indicates the loss of 
the metallicity of SWCNTs due to effective grafting [21] leading to a weakening of the coupling.
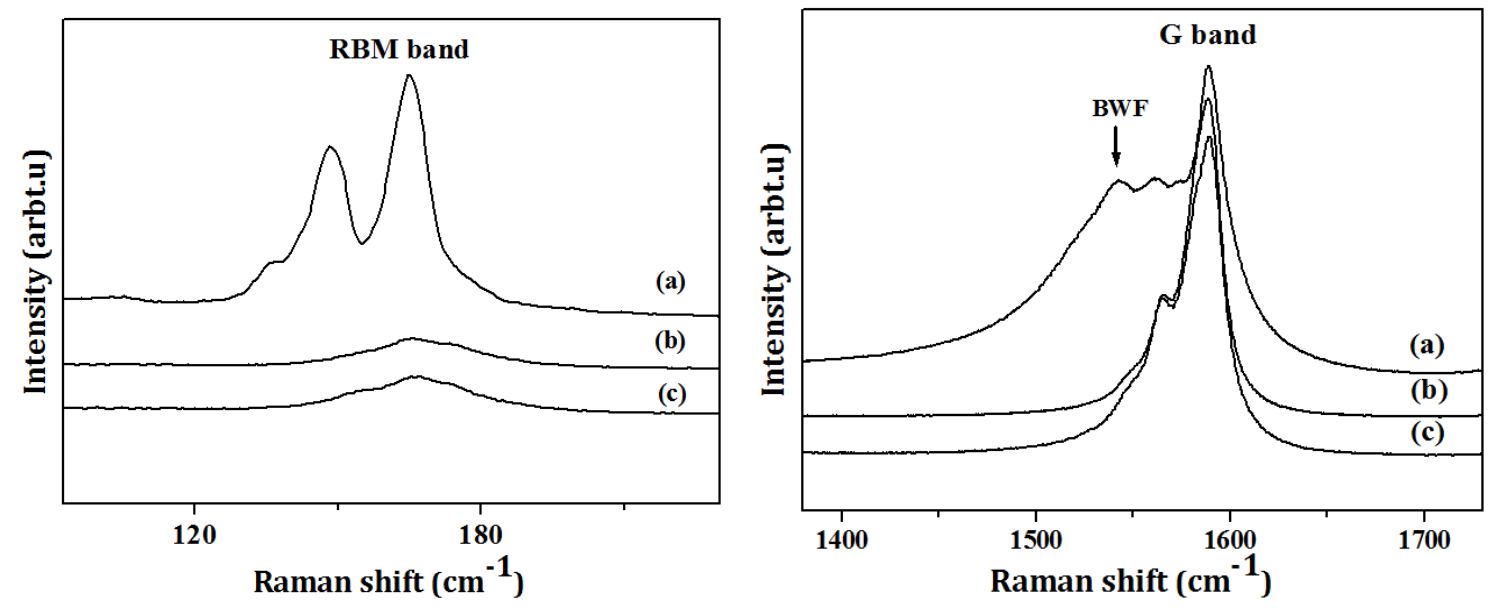

Figure 4: Raman spectrum ( $\lambda=633 \mathrm{~nm})$ of a) Raw SWCNTs, b) SWCNTs_PRG, and c) SWCNTs_CuHCF. The curves have been shifted in height for clarity.

For the 532nm and 488nm excitation wavelengths (Fig. 5 and 6), the resonance allows to probe the semiconductor nanotubes. Concerning the RBM region, a decrease of the peaks intensity is observed similarly to what has been described with the $633 \mathrm{~nm}$ excitation wavelength. This indicates that semiconducting tubes are also efficiently grafted. The $G$ bands of the SWCNTs_PRG and SWCNTs_CuHCF evolve the same way whatever the excitation energy (G band in Fig. 5 and 6). More precisely, the G band of SWCNTs_PRG shows a small shift of about $0.5 \mathrm{~cm}^{-1}$ towards low frequencies, which is attributed to the force constant modification between the carbon-carbon bonds following the PRG functionalization, which gives rise to an uniaxial or torsional strain [22]. The G band of the SWCNTs_CuHCF sample exhibits a shift of about $2 \mathrm{~cm}^{-1}$ towards high frequencies attributed to a p-type doping brought by the $\mathrm{HCF}$ nanoparticles [23].
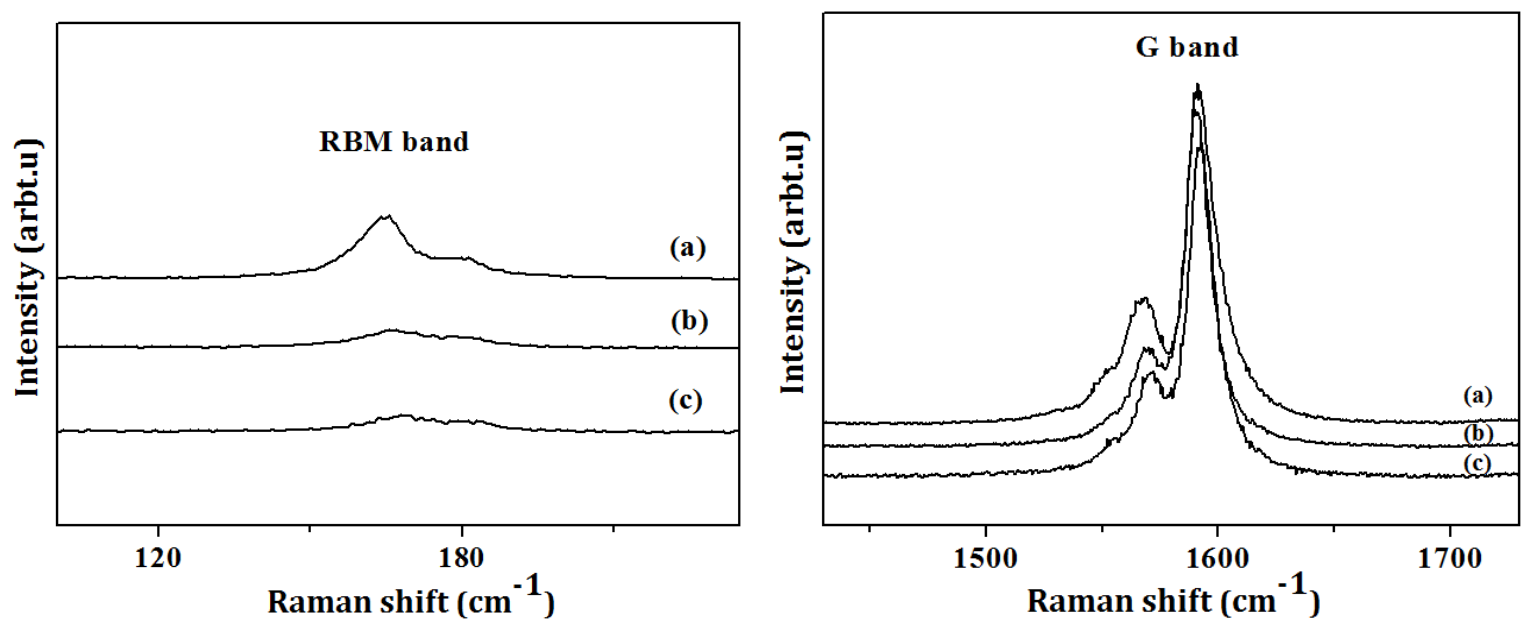

Figure 5: Raman spectrum ( $\lambda=488 \mathrm{~nm})$ of a) Raw SWCNTs, b) SWCNTs_PRG, and c) SWCNTs_CuHCF. The curves have been shifted in height for clarity. 

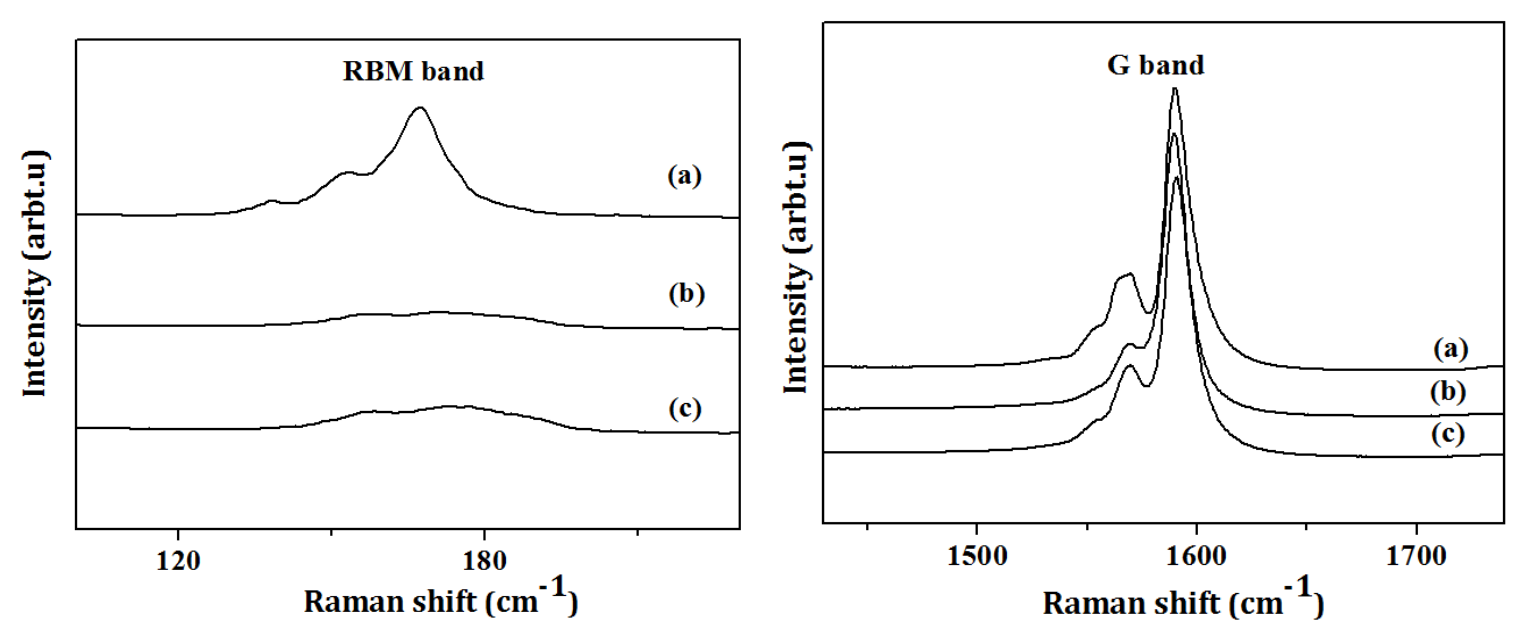

Figure 6: Raman spectrum ( $\lambda=532 \mathrm{~nm})$ of a) Raw SWCNTs, b) SWCNTs_PRG, and c) SWCNTs_CuHCF. The curves have been shifted in height for clarity.

\section{X-Ray Photoemission Spectroscopy}

XPS is an efficient technique to check the presence of organic groups, to identify the grafted molecules on the surface of SWCNTs and to reveal if they are covalently bound to the carbon walls. On the XPS survey of raw SWCNTs (Fig. 7.a), we identify, together with the expected carbon species around $285 \mathrm{eV}$, oxygen (around $530 \mathrm{eV}$ ), arising either from contamination and/or surface defects. The binding energy (BE) values for carbon and oxygen groups were attributed based on the reported literature [24-25]. On the PRG functionalized SWCNTs survey (Fig.7.b), in addition to carbon and oxygen, the presence of nitrogen is well identified, related to the presence of PRG grafted molecules. The growth of copper hexacyanoferrate-NPs $\mathrm{K}_{2} \mathrm{CuFe}(\mathrm{CN})_{6}$ is accompanied by identification of new components (Fig. 7.c) associated to the presence of the new elements which are potassium, copper and iron.

To better understand the nature of chemical bondings in the raw and functionalized samples, we performed a high resolution measurements. In the following, the number of contributions within the $\mathrm{C} 1 \mathrm{~s}$ peaks is kept constant, independently of the functionalization steps. Chemical modifications are thus evidenced by changes in peak FWHM. 


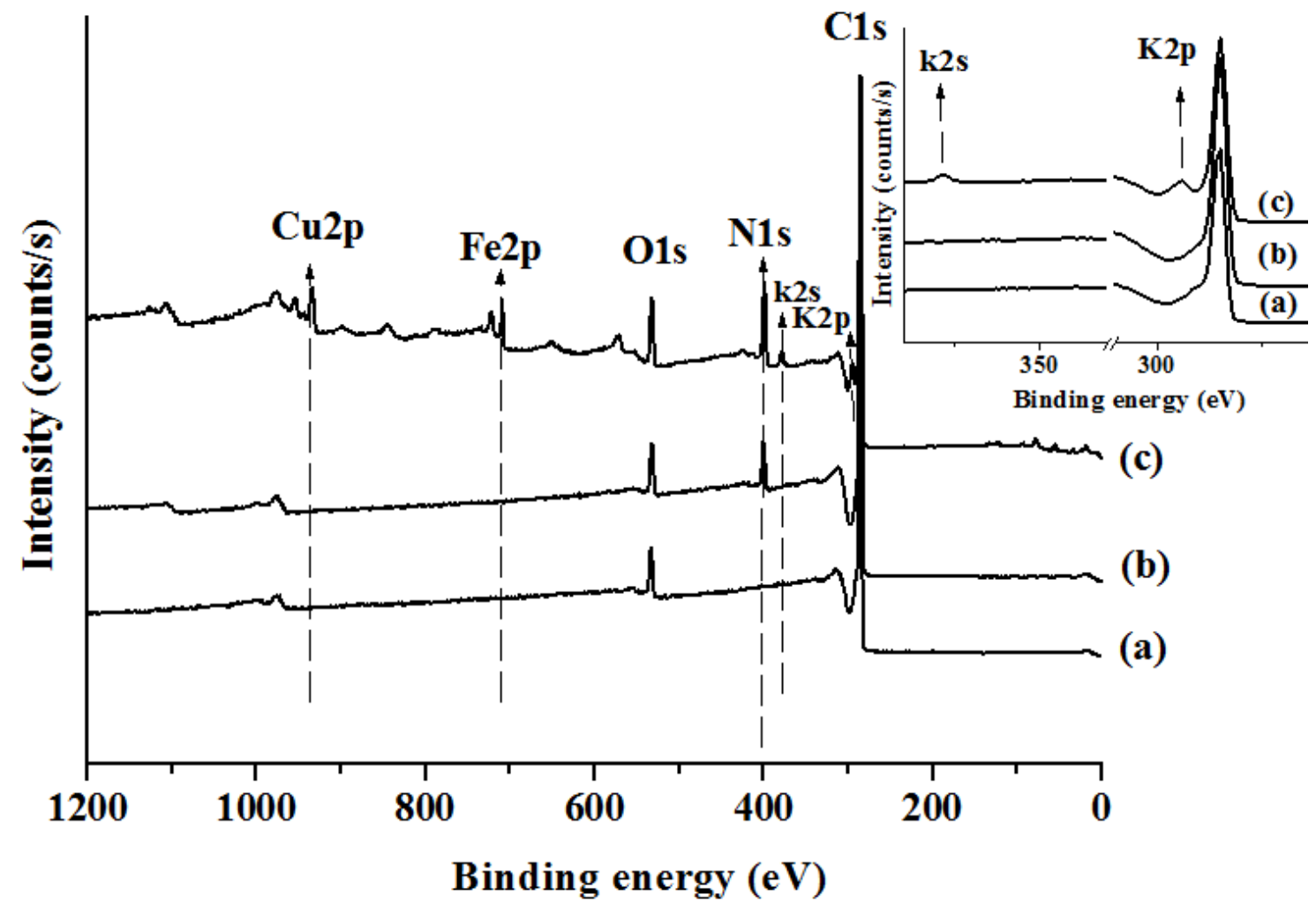

Figure 7: XPS surveys of a) Raw SWCNTs, b) SWCNTs_PRG and c) SWCNTs_CuHFC, The $\mathrm{C} 1 \mathrm{~s}$ edge of the raw sample (Fig. 8.a) is deconvoluted into six components (table 1):

\begin{tabular}{|c|c|c|c|c|c|}
\hline & BE $(\mathrm{eV})$ & FWHM $(\mathrm{eV})$ & Assignment & Atomic $\%$ & Ref \\
\hline Peak 1 & 283.5 & 0.9 & Vacancies & 2.7 & {$[25-26]$} \\
\hline Peak 2 & 284.4 & 0.70 & C=C & 77.3 & {$[24-25]$} \\
\hline Peak 3 & 285.2 & 1.0 & C-C/C-H & 11.6 & {$[24-25]$} \\
\hline Peak 4 & 286.2 & 1.0 & C-O & 3.9 & {$[24-25]$} \\
\hline Peak 5 & 287.1 & 1.0 & C=O & 0.7 & {$[24-25]$} \\
\hline Peak 6 & 288.7 & 2.4 & COOH & 3.8 & {$[24-25]$} \\
\hline
\end{tabular}

Table 1: Elemental ID and Quantification of C1s core level of raw SWCNTs_according to the XPS measurement

Comparing the C1s core level of raw SWCNTs with the PRG functionalized sample (Fig. 8.a and 8.b), we clearly observe modifications around $286.05 \mathrm{eV}$ (related to $\mathrm{C}-\mathrm{O}$ or $\mathrm{C}-\mathrm{N}$ bonds) [25] especially in terms of FWHM (from $1 \mathrm{eV}$ for raw SWCNTs to FWHM $=1.3 \mathrm{eV}$ for SWCNTs_PRG) and atomic content. This evolution is accompanied with a decrease of sp2 bonds at $284,4 \mathrm{eV}$ and an increase of $\mathrm{sp} 3$ bonds at $285 \mathrm{eV}$.

Indeed, the functionalization of SWCNTs with PRG is expected to result in an increase of the C-N bond contribution in XPS spectra, as well as in a simultaneous increase of the sp3 contribution and decrease of the sp2 contribution. The latter effect is linked to the cycloaddition mechanism between SWCNT and PRG. It appears here that the C-N bond contribution increase is larger than the intensity changes related to $\mathrm{C}-\mathrm{C}$ sp2 and sp3 bonds. This observation could 
be explained by the fact that a part of PRG adsorbed onto SWCNTs despite the acetone washing after grafting.

\begin{tabular}{|c|c|c|c|c|c|}
\hline & BE $(\mathrm{eV})$ & FWHM $(\mathrm{eV})$ & Assignment & Atomic $\%$ & Ref \\
\hline Peak 1 & 283.4 & 0.9 & Vacancies & 1.1 & {$[25-26]$} \\
\hline Peak 2 & 284.4 & 0.9 & C=C & 57.0 & {$[24-25]$} \\
\hline Peak 3 & 285.2 & 1.0 & C-C/C-H & 19.7 & {$[24-25]$} \\
\hline Peak 4 & 286.1 & 1.3 & C-O & 13.8 & {$[24-25]$} \\
\hline Peak 5 & 287.1 & 1.2 & $\mathrm{C}=\mathrm{O}$ & 3.5 & {$[24-25]$} \\
\hline Peak 6 & 288.77 & 2.40 & $\mathrm{COOH}$ & 4.9 & {$[24-25]$} \\
\hline
\end{tabular}

Table 2: Elemental ID and Quantification of C1s core level of SWCNTs_PRG_according to the XPS measurements

\begin{tabular}{|c|c|c|c|c|c|}
\hline & BE $(\mathrm{eV})$ & FWHM $(\mathrm{eV})$ & Assignment & Atomic $\%$ & Ref \\
\hline Peak 1 & 283.3 & 0.9 & Vacancies & 1.1 & {$[25-26]$} \\
\hline Peak 2 & 284.4 & 0.9 & C=C & 64.1 & {$[24-25]$} \\
\hline Peak 3 & 285.3 & 1.1 & C-C/C-H & 16.1 & {$[24-25]$} \\
\hline Peak 4 & 286.2 & 1.2 & C-O & 10.6 & {$[24-25]$} \\
\hline Peak 5 & 287.4 & 1.5 & C=O & 3.4 & {$[24-25]$} \\
\hline Peak 6 & 289.2 & 2.6 & COOH & 4.7 & {$[24-25]$} \\
\hline
\end{tabular}

Table 3: Elemental ID and Quantification of C1s core level of SWCNTs_CuHCF_according to the XPS measurements

For CuHCF functionnalized SWCNTs (figure 8.c), the signal around $286 \mathrm{eV}$ related to C-N bonds decreases compared to SWCNTs_PRG from $\sim 14 \%$ to $10.6 \%$. Considering that the sample is washed again after the total functionalization, i.e. after the growth of hexacyanoferrate nanoparticles, PRG-CuHCF particles not covalently bound to SWCNTs could have been removed during this final washing step.

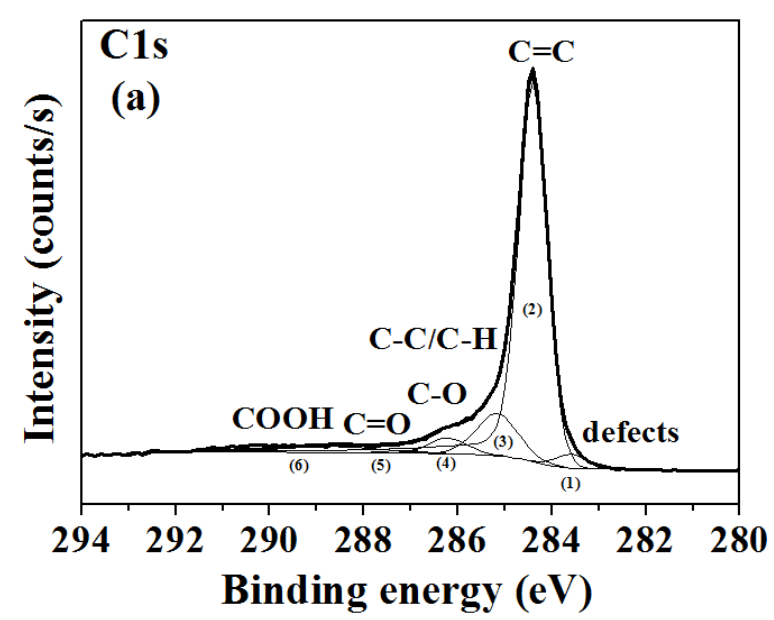




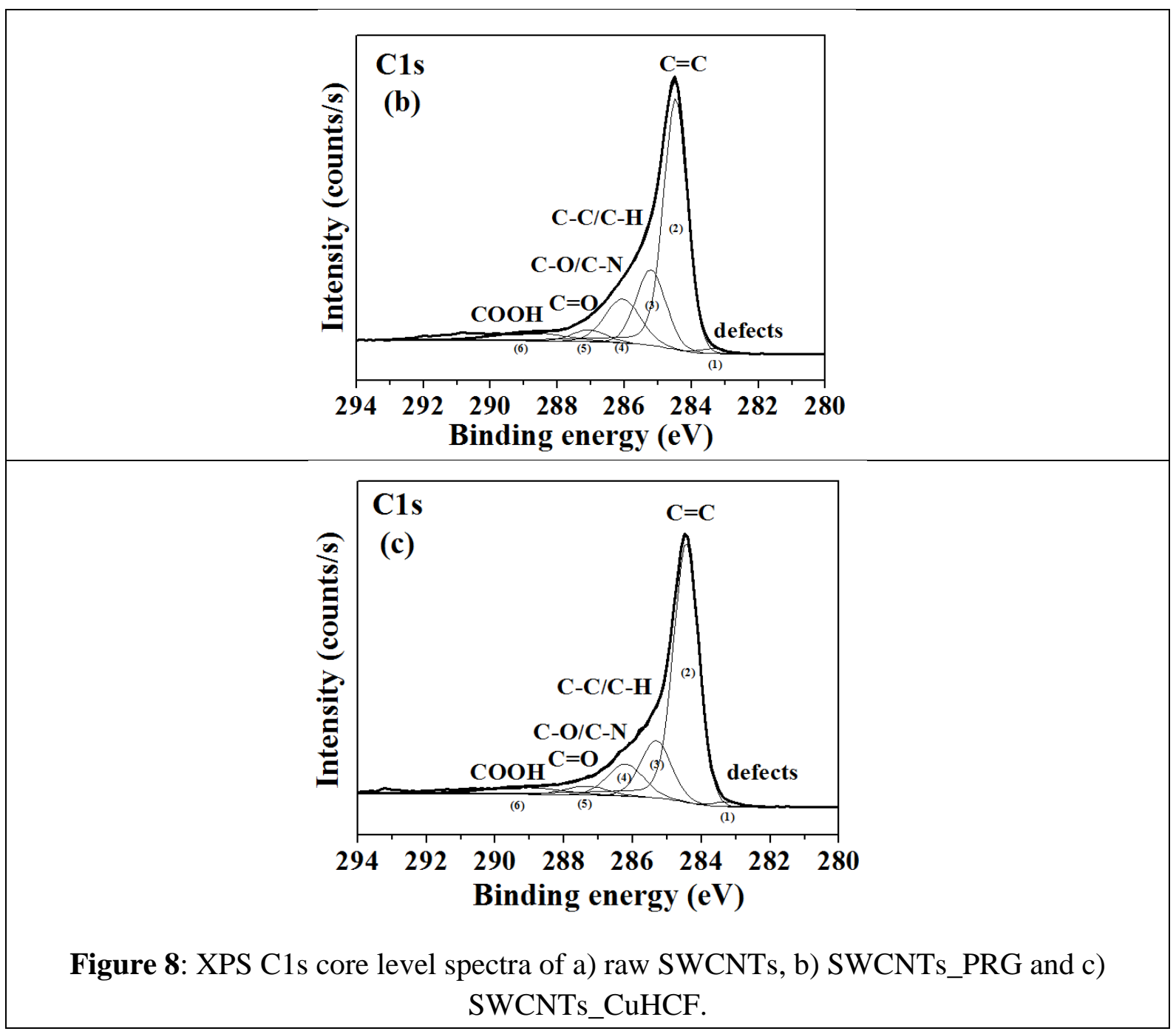

The typical BE for nitrogen atoms in cyanide groups in hexacyanoferrate can be found between $397.5 \mathrm{eV}$ and 400eV [27-29]. Figures 9.a and 9.b display the high resolution N1s spectra of the SWCNTs_PRG and SWCNTs_CuHCF samples. The most affected N1s signal following the growth of CuHCF_NPs is at $397.6 \mathrm{eV}$, which shows a drastic intensity increase, so that we assign this signal to $\mathrm{C} \equiv \mathrm{N}$ bonds in $\mathrm{CuHCF}$ _NPs. Thus, the nitrogen N1s is deconvoluted into four peaks involving $\mathrm{C} \equiv \mathrm{N}(397.6 \mathrm{eV}), \mathrm{C}-\mathrm{NH}_{2}(399 \mathrm{eV}), \mathrm{N}-\mathrm{C}=\mathrm{O}(400.2 \mathrm{eV})$ and $\mathrm{N}^{+}(401.8 \mathrm{eV})$ bonds [30-31].

In the $\mathrm{Cu} 2 \mathrm{p}$ and $\mathrm{Fe} 2 \mathrm{p}$ XPS core level spectra of CuHFC functionalized SWCNT (Fig. 9.c and $\mathrm{d}$ ), we identify the presence of a bond at $932.7 \mathrm{eV}$ corresponding to $\mathrm{Cu} 2 \mathrm{p}$, and a bond at $708.31 \mathrm{eV}$ attributed to Fe2p [32]. Biesinger et al [32] have analysed different types of copper samples such as $\mathrm{Cu}$ metal and $\mathrm{Cu}_{2} \mathrm{O}$ and found the same deconvolution profile of $\mathrm{Cu} 2 \mathrm{p}$ as in our sample. They have attributed the bond at lower energy to $\mathrm{Cu}^{+}$and the one at higher energy to $\mathrm{Cu}^{2+}$. In these compounds, $\mathrm{Cu} 2+$ is generally expected [33-34] but a reversible solid-state reduction to $\mathrm{Cu}^{+}$is sometimes reported [35].

It is worth mentioning that the ratio $\mathrm{Cu}^{+} / \mathrm{Cu}^{2+}$ is strongly dependent on the area under investigation in our samples. As we do not believe in a sample heterogeneity, we assume a 
reduction under x-ray beam, leading to a transformation of $\mathrm{Cu}^{2+}$ into $\mathrm{Cu}^{+}$. This point will be accurately studied in the next investigations.
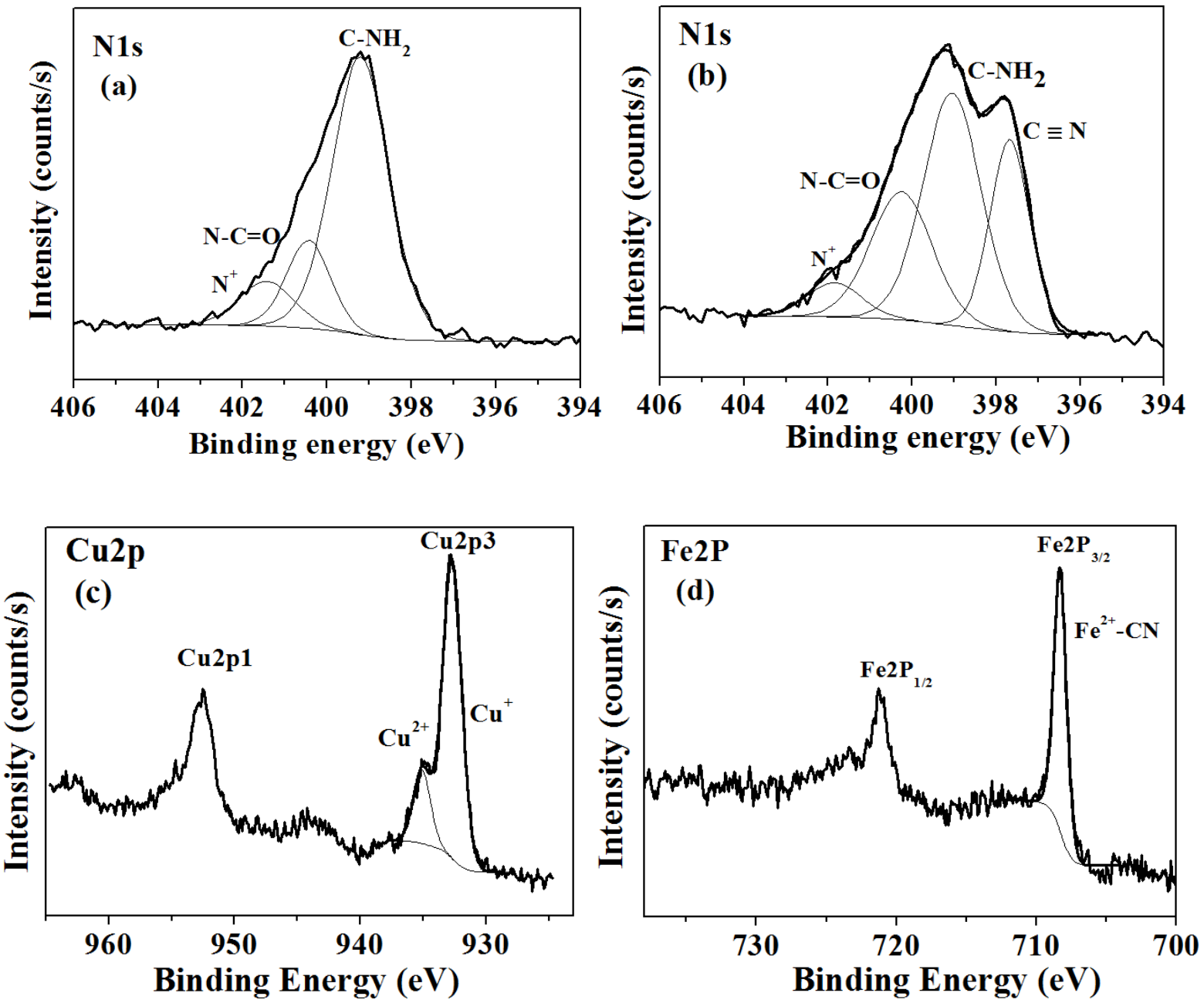

Figure 9: XPS N1s core level spectra of a) SWCNTs_PRG and b) SWCNTs_CuHCF c) XPS $\mathrm{Cu} 2 \mathrm{p}$ core level spectra of CuHCF_SWCNTs, d) XPS Fe2pcore level spectra of CuHCF_SWCNTs

\section{Cs sorption batch studies}

The SWCNTs are used as CuHCF functionalized substrates for cesium capture. To evaluate the rate of $\mathrm{Cs}^{+}$sorption on raw and functionalized SWCNTs, we performed sorption experiments by exposing the samples to $\mathrm{CsNO}_{3}$ solution. We have firstly characterized samples exposed to the cesium solution by XPS analysis in order to reveal the presence of cesium and to check the evolution of the sorption capacity versus $\mathrm{Cs}^{+}$concentration.

The full surveys of unexposed CuHCF functionalized SWCNTs and of CuHCF functionalized SWCNTs after exposure to cesium nitrate solutions of different concentrations are presented in Fig. 10. The signal of the $\mathrm{Cs} 3 \mathrm{p}_{3 / 2}$ and $\mathrm{Cs} 3 \mathrm{~d}_{5 / 2}$ are detected respectively at $\mathrm{BE} \sim 997 \mathrm{eV}$ and $\sim 724 \mathrm{eV}$ (Fig. 10). The intensity of each of these peaks increases with the increase of the cesium 
nitrate concentration (inset in Fig. 10). On the other hand, we note, as expected, the disappearance of the signal coming from potassium upon cesium exposure, which is in accordance with the exchange between $\mathrm{Cs}^{+}$and $\mathrm{K}^{+}$ions initially contained in CuHCF_NPs.

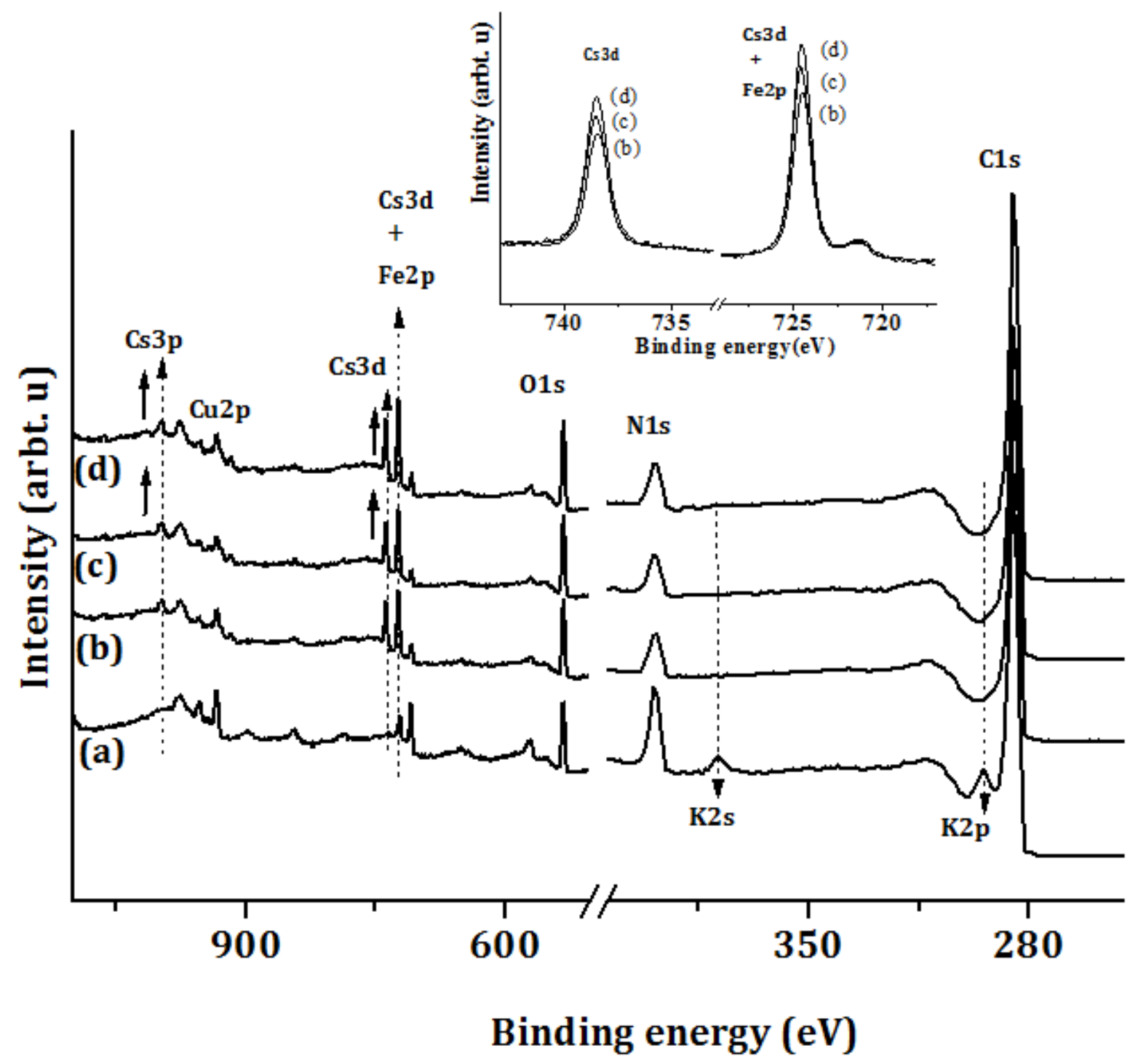

Figure 10: XPS surveys of a) SWCNTs_CuHCF, b) SWCNTs_CuHCF_Cs [0.1mM], c) SWCNTs_CuHCF_Cs [2mM], and d)SWCNTs_CuHCF_Cs [4mM] (inset: XPS of Cs3d core levels).

In order to go further in the understanding of the sorption mechanism in this sample, we performed LPIC analyses of the solution before and after exposure to SWCNTs_CuHCF.

We measured the sorption isotherm of both raw and CuHCF functionalized SWCNTs samples from LPIC of the solutions, using the sorption capacity $\Phi_{a d s}\left(\mathrm{mg}_{\mathrm{g}} \mathrm{g}^{-1}\right)$ defined as:

$$
\Phi_{a d s}=\left(C_{i}-C_{f}\right) \frac{V}{m}
$$


$C_{i}$ is the initial mass concentration $\left(\mathrm{mg} . \mathrm{L}^{-1}\right)$ of $\mathrm{Cs}^{+}$in the $\mathrm{CsNO}_{3}$ solution. $C_{f}$ is the final mass concentration (mg.L $\mathrm{L}^{-1}$ ) of $\mathrm{Cs}^{+}$in the $\mathrm{CsNO}_{3}$ solution, $V$ is the volume of the $\mathrm{CsNO}_{3}$ solution (L) and $m$ is the substrate mass $(\mathrm{g})$.

The sorption isotherm of $\mathrm{Cs}^{+}$on raw and CuHCF functionalized SWCNTs are illustrated in Fig.11. Both raw and CuHCF functionalized SWCNTs show an increasing sorption capacity with $\mathrm{Cs}^{+}$concentration, though it is always lower for raw SWCNTs (up to $150 \mathrm{mg} \cdot \mathrm{g}^{-1}$ for raw SWCNTs and to $230 \mathrm{mg} \cdot \mathrm{g}^{-1}$ for CuHCF_SWCNTs). A coarse way of evaluating the proportion of $\mathrm{Cs}^{+}$ions complexed in $\mathrm{CuHCF}_{-} \mathrm{NPs}$ consists in subtracting the sorption isotherm of raw SWCNTs to that of SWCNTs_CuHCF. Therefore, the subtraction result (squares in Fig. 11) should correspond to the Cs selective part of the sorption isotherm. We found that the selective sorption of Cs reaches a plateau at a solution concentration of $4 \mathrm{mM}$, whose value is of about $80 \mathrm{mg} \cdot \mathrm{g}^{-1}$.

It is very interesting to remark that this value compares very well with the value obtained on the basis of XPS measurements. The fact that only the Cs fraction contained in the CuHCF_NPs is detected by XPS could be related to the degasing needed by the latter technique, which could be responsible for the removal of Cs ions bound to the SWCNT surface, and not intercalated inside CuHCF_NPs.

This sorption capacity can be compared with that of other materials dedicated to Cs removal from aqueous effluents. MWCNTs-reinforced zeolite-A beads have been developed for the removal of cesium and strontium from water and were studied in a recent work showing that the maximum theoretical sorption capacity for cesium was $113 \mathrm{mg} \cdot \mathrm{g}^{-1}$ [7]. Oxidized MWCNTs were also used for cesium capture and it have been demonstrated that they can sorb a maximum value of about $10 \mathrm{mg} \cdot \mathrm{g}^{-1}$. This low sorption capacity is related to their multiwall structure that limits their specific surface and therefore reduces the sorption capacity [5]. Other studies showed that SWCNTs buckypapers can be functionalized with DMAD and are able to sorb about 250mg. $\mathrm{g}^{-1}$, but DMAD molecules are not selective of Cs [9]. The sorption capacity found for SWCNTs_CuHCF substrates is therefore very encouraging. For pure CuHCF, a maximum Cs sorption capacity of $420 \mathrm{mg} \cdot \mathrm{g}^{-1}$ has been determined [36]. Provided that the mass content of CuHCF_NPs is 15 wt.\%, we can conclude that all available sites in our CuHCF_NPs are occupied by $\mathrm{Cs}^{+}$ions. This is in very good agreement with the disappearance of the $\mathrm{K}$ peak in the XPS survey. 


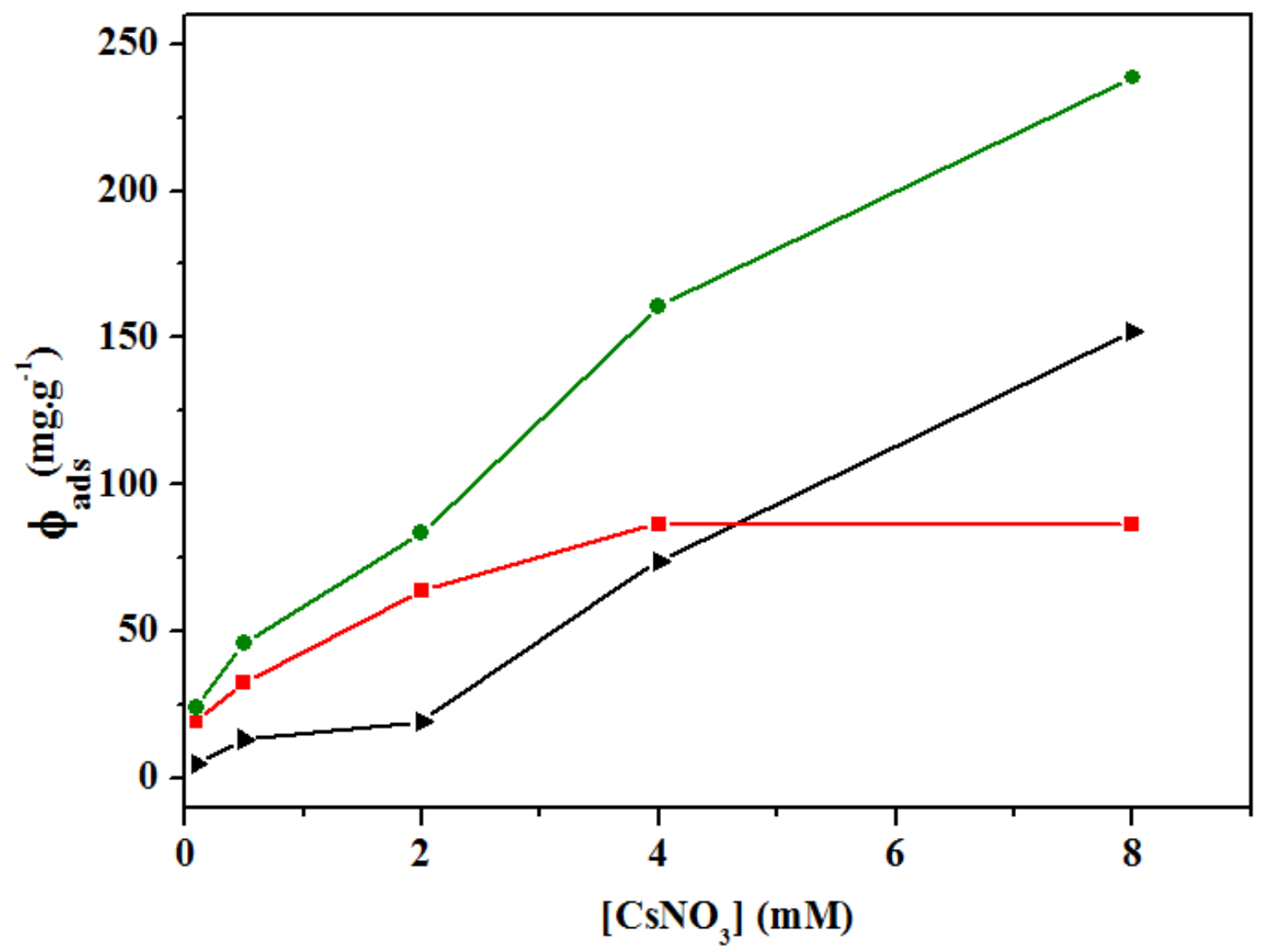

Figure 11: Sorption isotherm of $\mathrm{Cs}^{+}$ions on: raw SWCNTs and SWCNTs_CuHCF. $\mathrm{Cs}^{+}$ions selectively sorbed inside CuHCF_NPs.

\section{Conclusion}

We reported on the elaboration and characterization of new sorbent materials for the solidliquid extraction of $\mathrm{Cs}^{+}$ions. Propargylamine molecules are first grafted onto the SWCNT surface and act as a linker for the growth of CuHCF nanoparticles. A high grafting rate of PRG ( 1 molecule for 30 carbon atoms) results in $15 \mathrm{wt} . \%$ of CuHCF nanoparticles in the end of the elaboration process. Based on a careful analysis combining XPS of the materials after Cs exposure, and LPIC of the solutions before and after exposure, it was possible to determine a total sorption capacity of $230 \mathrm{mg} \cdot \mathrm{g}^{-1}$ of $\mathrm{Cs}^{+}$ions, among which $80 \mathrm{mg} \cdot \mathrm{g}^{-1}$ are likely to be selectively incorporated inside CuHCF_NPs. These results compare nicely with existing solid substrates dedicated to Cs capture, and open interesting perspectives towards their integration inside electrodriven devices for the controlled sorption/desorption of $\mathrm{Cs}^{+}$ions.

\section{Acknowledgements}


The authors acknowledge financial support from XXX and the assistance from the Balard platform in Montpellier.

Support financier: CEA, Université de Tunis et L2C

Discussions scientifiques et assistance technique :

Raman : Alban Jonchere, David Maurin

LPIC : Cyrielle Rey

MET : Henri Pierre Brau et Xavier Le Goff 


\section{References}

(1) Jang, S. C.; Hong, S. B.; Yang, H. M.; Lee, K. W.; Moon, J. K.; Seo, B. K.; Huh, Y. S.; Roh, C. Removal of Radioactive Cesium Using Prussian Blue Magnetic Nanoparticles. Nanomaterials. 2014, 4, 894-901.

(2) Zhang, H.; Zhao, X.; Wei, J.; Li, F. Removal of cesium from low-level radioactive wastewaters using magnetic potassium titanium hexacyanoferrate. Chem. Eng. Journal. 2015, 275, 262-270.

(3) Causse, J.; Tokarev, A.; Ravaux, J.; Moloney, M.; Barré, Y.; Grandjean, A.; Facile onepot synthesis of copper hexacyanoferrate nanoparticle functionalized silica monolths for the selective entrapment of ${ }^{137}$ Cs. J. Mater. Chem.A. 2014, 2, 9461-9464.

(4) Delchet, C.; Tokarev, A.; Dumail, X.; Toquer, G.; Barré, Y.; Guari, Y.; Guerin, C.; Larionova, J. ; Grandjean, A. Extraction of radioactive cesium using innovative functionalized porous materials. $R S C A d v$. 2012, 2, 5707-5716.

(5) Yavari, R.; Huang, Y. D.; Ahmadi, S. J. Adsorption of cesium(I) drom aqueous solution using oxidized multiwall carbon nanotube. J. Rad. Nucl. Chem. 2011, 287, 393-401.

(6) Vipin, A. K.; Ling, S.; Fugetsu, B. Sodium cobalt hexacyanoferrate encapsulated in alginate vesicle with CNT for both cesium and strontium removal. Carb. Polym. 2014, 111, 477-484.

(7) Vipin, A. K.; Ling, S.; Fugetsu, B. Removal of $\mathrm{Cs}^{+}$and $\mathrm{Sr}^{+}$from water using MWCNT reinforced Zeolite-A beads. Micro. Meso. Mat. 2016, 224, 84-88.

(8) Krawczyk, M.; Skowron, M. J. Multiwalled carbon nanotubes as solid sorbent in dispersive micro solid-phase extraction for sequential determination of cadmium and lead in water samples. Microchemical Journal. 2016, 126, 296-301.

(9) Kaper, H.; Nicolle, J.; Cambedouzou, J.; Grandjean, A. Multi-method analysis of functionalized single-walled carbon nanotubes for cesium liquid-solid extraction. Mater. Chem. Phys. 2014, 147, 147-154.

(10) Li, N.; He, B; Xu, S.; Yuan, J.; Miao, J.; Niu, L.; Song, J. In site formation and growth of Prussian blue nanoparticles anchored to multiwalled carbon nanotubes with poly(4vinylpyridine) linker by layer-by-layer assembly. Mater. Chem. Phys. 2012, 133, 726-734. 
(11) Lee, H.K.; Choi, J. W.; Oh, W.; Choi, S. J. Sorption of cesium ions from aqueous solutions by multi-walled carbon nanotubes functionalized with copper ferrocyanide. J. Radioanal. Nucl. Chem. 2016, 309, 477-484.

(12) Nicolle, J.; Le Goff , X.; Grandjean, A.; Cambedouzou, J. Observing and predicting the preferential functionalization of metallic or semiconducting single-walled carbon nanotubes. Europhysics Letters. 2014, 107, 67003.

(13) Grandjean, A.; Delchet, C.; Causse, J.; Barré, Y.; Guari, Y.; Larionova, J. Effect of the chemical nature of different transition metal ferrocyanides to entrap Cs. J. Radioanal. Nucl. Chem. 2016, 307, 427-436.

(14) Shirley, D. A. High-Resolution X-Ray Photoemission Spectrum of the Valence Bands of Gold. Phys. Rev. B. 1972, 5, 4709.

(15) Scofield, J. H. Hartree-Slater subshell photoionization cross-sections at 1254 and $1487 \mathrm{eV}$. J. Electron. Spectrosc. Relat. Phenom. 1976, 8, 129-137.

(16) Vigolo, B.; Mamane, V.; Valsaque, F.; Ha. Le, T. N.; Thabit, J. ; Ghanbaja, J.; Aranda, L.; Fort, Y.; McRae, E. Evidence of sidewall covalent functionalization of single-walled carbon nanotubes and its advantages for composite processing. Carbon. 2009, 47, 411-416.

(17) Munirasu, S.; Albuerne, J.; Boschetti-de-Fierro, A.; Abetz, V. Functionalization of carbon materials using the Diels-Alder reaction. Macromol. Rapid Commun. 2010, 31, 574-579.

(18) Hussain, S.; Jha, P.; Chouksey, A.; Raman, R.; Islam, S. S.; Islam, T.; Choudhary, P. K. Spectroscopic Investigation of Modified Single Wall Carbon Nanotube (SWCNTs). Journal of Modern Physics. 2011, 2, 538-543.

(19) Liu, R. The Functionalization of Carbon Nanotubes, Ph.D. Thesis, School of Chemistry, University of NSW, Sydney, 2008.

(20) Piscanec, S.; Lazzeri, M.; Robertson, J.; Ferrari, A. C.; F. Mauri. Optical phonons in carbon nanotubes: Kohn anomalies, Peierls distortions, and dynamic effects. Phys. Rev. Lett. 2007, B75, 035427.

(21) Paillet, M.; Poncharal, P.; Zahab, A.; Sauvajol, J.L.; Meyer, J. C.; Roth, S. Vanishing of the Breit-Wigner-Fano component in individual single-wall carbon nanotubes. Phys. Rev. Lett. 2005, 94(237401), 1-4.

(22) Liu, Z.; Zhang, J.; Gao, B. Raman spectroscopy of strained single-walled carbon nanotubes. Chem. Commun. 2009, 10.1039, 6902-6918. 
(23) Forment-Aliaga, A.; Weitz, R. T.; Sagar, A. S.; Lee, E. J. H; Konuma, M; Burghard, M.; Kern, K. Strong p-Type Doping of Individual Carbon Nanotubes by Prussian Blue Functionalization. Small. 2008, 4(10), 1671-1675.

(24) Ling, X.; Wei, Y.; Zou. L.; Xu, S. The effect of different order of purification treatment on the purity of multiwalled carbon nanotubes. Appl. Surf. Sci. 2013, 276, 159-166.

(25) Ramirez-Calera, I. J.; Meza-Laguna, V.; Gromovoy, T. Yu.; Chavez-Uribe, M. I.; Basiuk, V. A.; Basiuk, E. V.; Solvent-free functionalization of fullerene C60 and pristine multi-walled carbon nanotubes with aromatic amines. Appl. Surf. Sci. 2015, 328, 45-62.

(26) Yamada, Y.; Yasuda, H.; Murota, K.; Nakamura, M.; Sodesawa, T.; Sato, S. Analysis of heat-treated graphite oxide by X-ray photoelectron Spectroscopy. J. Mater. Sci. 2013, 48, 8171-8198.

(27) Datta, M.; Datta, A. In situ FTIR and XPS studies of the hexacyanoferrate redox system. J. Phys. Chem. 1990, 94(21), 8203-8207.

(28) Malik, M.A.; Kulesza, P.J.; Włodarczyk, R.; Wittstock, G.; Szargan, R.; Bala, H.; Galus, Z. Formation of ultra-thin prussian blue layer on carbon steel that promotes adherence of hybrid polypyrrole based protective coating. J. Solid State Electrochem. 2005, 9, 403-411.

(29) Naumkin, A. V.; Kraut-Vass, A.; Gaarenstroom, S. W.; Powell, C. J. NIST X-ray Photoelectron Spectroscopy Database. The U.S. Secretary of Commerce on behalf of the United States of America, 2000 http://srdata.nist.gov/xps/Default.aspx.

(30) Jia-lin, Y.; Gui-jiao, C.; Jun, C.; Wei, Y.; Bang-hu, X.; Ming-bo, Y. Functionalized graphene oxide with ethlenediamine and 1, 6-hexanediamine. New Carbon Materials.2012, 27(5), 370-376.

(31) Ren, Z.; Xu, X.; Wang, X.; Gao, B.; Yue, Q.; Song, W.; Zhang, L.; Wang, H. FTIR, Raman, and XPS analysis during phosphate, nitrate and Cr(VI) removal by amine cross-linking biosorbent. J. Colloid Interface Sci. 2016, 468, 313-323.

(32) Biesinger, M. C.; Lau, L. W.M.; Gerson, A. R.; Smart, R. S.C. Resolving surface chemical states in XPS analysis of first row transition metals, oxides and hydroxides: $\mathrm{Sc}, \mathrm{Ti}, \mathrm{V}$, $\mathrm{Cu}$ and Zn. App. Sur. Sci. 2010, 257, 887-898.

(33) Wessells, C. D.; Huggins, R. A.; Cui, Y. Copper hexacyanoferrate battery electrodes with long cycle life and high power. Nature Comm 2. 2011, 550, 10.1038.

(34) Lisowska-Oleksiak, A.; Wilamowska, M.; Jasulaitiené, V. Organic-inorganic composites consisted of poly(3,4-ethylenedioxythiophene) and Prussian Blue analogues. Electrochimica. Acta. 2011, 56, 3626-3632. 
(35) Oktawian, M.; Jadwiga, S.; Pawel -J, K.; Marcin-A, M.; Zbigniew, G. Electrochemical identity of copper hexacyanoferrate in the solid-state: evidence for the presence and redox activity of both iron and copper ionic sites. J. Elect. Chem. 2002, 532, 157-164.

(36) Omura, A.; Moritomo, Y. Cs ${ }^{+}$trapping in size-controlled nanopaves of hexacyanoferrates. App. Phys. Express. 2012, 5(057101), 1-3. 